\title{
Dust mass distribution around comet 67P/Churyumov-Gerasimenko determined via parallax measurements using Rosetta's OSIRIS cameras
}

\author{
T. Ott, ${ }^{1 \star} \dagger$ E. Drolshagen, ${ }^{1 \star} \dagger$ D. Koschny, ${ }^{2,3}$ C. Güttler, ${ }^{4}$ C. Tubiana, ${ }^{4}$ E. Frattin,,${ }^{5,6}$ \\ J. Agarwal, ${ }^{4}$ H. Sierks, ${ }^{4}$ I. Bertini, ${ }^{6}$ C. Barbieri, ${ }^{7}$ P. I. Lamy,${ }^{8}$ R. Rodrigo, $,{ }^{9}, 10$ \\ H. Rickman, ${ }^{11,12}$ M. F. A'Hearn, ${ }^{4,13,14}$ M. A. Barucci, ${ }^{15}$ J.-L. Bertaux, ${ }^{16}$ \\ S. Boudreault, ${ }^{4}$ G. Cremonese, ${ }^{5}$ V. Da Deppo, ${ }^{17}$ B. Davidsson, ${ }^{14}$ S. Debei, ${ }^{18}$ \\ M. De Cecco, ${ }^{19}$ J. Deller, ${ }^{4}$ C. Feller,${ }^{15}$ S. Fornasier, ${ }^{15}$ M. Fulle, ${ }^{20}$ B. Geiger,${ }^{21}$
}

A. Gicquel, ${ }^{4}$ O. Groussin, ${ }^{22}$ P. J. Gutiérrez, ${ }^{23}$ M. Hofmann, ${ }^{4}$ S. F. Hviid, ${ }^{24}$

W.-H. Ip, ${ }^{25,26}$ L. Jorda, ${ }^{22}$ H. U. Keller, ${ }^{24,27}$ J. Knollenberg, ${ }^{24}$ G. Kovacs, ${ }^{4}$

J. R. Kramm, ${ }^{4}$ E. Kührt, ${ }^{24}$ M. Küppers, ${ }^{21}$ L. M. Lara, ${ }^{23}$ M. Lazzarin, ${ }^{7}$ Z.-Y. Lin, ${ }^{25}$

J. J. López-Moreno, ${ }^{23}$ F. Marzari, ${ }^{7}$ S. Mottola,${ }^{24}$ G. Naletto, ${ }^{17,28,29}$ N. Oklay, ${ }^{24}$

M. Pajola, ${ }^{30}$ X. Shi, ${ }^{4}$ N. Thomas, ${ }^{31,32}$ J.-B. Vincent ${ }^{24}$ and B. Poppe ${ }^{1}$

Affiliations are listed at the end of the paper

Accepted 2017 June 3. Received 2017 June 1; in original form 2017 March 31

\begin{abstract}
The OSIRIS (optical, spectroscopic and infrared remote imaging system) instrument on board the ESA Rosetta spacecraft collected data of 67P/Churyumov-Gerasimenko for over $2 \mathrm{yr}$. OSIRIS consists of two cameras, a Narrow Angle Camera and a Wide Angle Camera. For specific imaging sequences related to the observation of dust aggregates in 67P's coma, the two cameras were operating simultaneously. The two cameras are mounted $0.7 \mathrm{~m}$ apart from each other, as a result this baseline yields a parallax shift of the apparent particle trails on the analysed images directly proportional to their distance. Thanks to such shifts, the distance between observed dust aggregates and the spacecraft was determined. This method works for particles closer than $6000 \mathrm{~m}$ to the spacecraft and requires very few assumptions. We found over 250 particles in a suitable distance range with sizes of some centimetres, masses in the range of $10^{-6}-10^{2} \mathrm{~kg}$ and a mean velocity of about $2.4 \mathrm{~m} \mathrm{~s}^{-1}$ relative to the nucleus. Furthermore, the spectral slope was analysed showing a decrease in the median spectral slope of the particles with time. The further a particle is from the spacecraft the fainter is its signal. For this reason, this was counterbalanced by a debiasing. Moreover, the dust mass-loss rate of the nucleus could be computed as well as the $A f \rho$ of the comet around perihelion. The summed-up dust mass-loss rate for the mass bins $10^{-4}-10^{2} \mathrm{~kg}$ is almost $8300 \mathrm{~kg} \mathrm{~s}^{-1}$.
\end{abstract}

Key words: techniques: image processing-parallaxes-comets: general-comets: individual: 67P/Churyumov-Gerasimenko.

\section{INTRODUCTION}

The ESA Rosetta mission was launched in 2004 and arrived at comet 67P/Churyumov-Gerasimenko (67P) in 2014. The spacecraft escorted the Jupiter-family comet for over $2 \mathrm{yr}$ in close vicinity. 67P

\footnotetext{
^E-mail: Theresa.Ott@uol.de (TO); Esther.Drolshagen@uol.de (ED)

$\dagger$ These authors contributed equally to this work.
}

reached its perihelion on 2015 August 13. The scientific camera system on board Rosetta is called OSIRIS (Optical, Spectroscopic and Infrared Remote Imaging System). It consists of two cameras with different fields of view (FOV). One is the Narrow Angle Camera (NAC) with an FOV of $2.20^{\circ} \times 2.22^{\circ}$. The other one is the Wide Angle Camera (WAC) with an FOV of $11.35^{\circ} \times 12.11^{\circ}$. For more information about OSIRIS, see Keller et al. (2007).

By means of the OSIRIS observations, it is possible to determine the mass of the particles around the nucleus. In order to do so, 
Table 1. The data sets analysed. The date of the observation, the name of the STP (short-term planning) directory and of the parent folder. The number of image sets in which we found particles with determinable distances to the spacecraft as well as the number of those particles. The WAC exposure time is 64 or $90 \mathrm{~s}$. When the WAC was exposed for $64 \mathrm{~s}$ the NAC took three images, and when the WAC was exposed for $90 \mathrm{~s}$ the NAC took four images. Moreover, the phase angle at which the data were recorded is listed. In some image sequences the phase angle was constant and in other, dedicated to examine the phase function, it changes in steps of $10^{\circ}$. Additionally, the distance from Rosetta to the nucleus is shown for each observation day.

\begin{tabular}{|c|c|c|c|c|c|c|c|c|}
\hline Date & STP & MTP & $\begin{array}{c}\text { \# usable } \\
\text { sets }\end{array}$ & $\begin{array}{l}\text { \# usable } \\
\text { particles }\end{array}$ & $\begin{array}{c}\text { WAC } \\
\text { exposure } \\
\text { time (s) }\end{array}$ & Perihelion & $\begin{array}{c}\text { Phase } \\
\text { angle } \\
\left({ }^{\circ}\right)\end{array}$ & $\begin{array}{c}\text { Distance } \\
\text { to nucleus } \\
{[\mathrm{m}]}\end{array}$ \\
\hline 03-8-15 & STP067_GRAIN _COLOR_001 & MTP19P & 9 & 69 & 64 & Pre & $79.4-149.4$ & 211000 \\
\hline $04-8-15$ & STP067_GRAIN _COLOR_002 & MTP19P & 9 & 86 & 64 & Pre & 44.4 & 238000 \\
\hline $25-8-15$ & STP070_GRAIN _COLOR_003 & MTP19P & 7 & 40 & 90 & Post & $75.3-115.2$ & 401000 \\
\hline $11-9-15$ & STP073_GRAIN _COLOR_001 & MTP20P & 3 & 24 & 90 & Post & 29.0 & 328000 \\
\hline 28-9-15 & STP075_GRAIN_COLOR_002 & MTP21P & 1 & 2 & 90 & Post & 114.6 & 1216000 \\
\hline Total & & & 40 & 262 & 3028 & & & \\
\hline
\end{tabular}

Table 2. The utilized filters. The WAC took all images with the green filter, the F21. The NAC collected data with different filters, the order in that the images were acquired is: F41, (F32 after perihelion), F22 and F24. The information is taken from Keller et al. (2007).

\begin{tabular}{cccc}
\hline Filter number & Central wavelength $(\mathrm{nm})$ & Filter name & Camera \\
\hline 41 & 882.1 & Near-IR_FFP-IR & NAC \\
32 & 649.2 & NFP-Vis_Orange & NAC \\
22 & 649.2 & FFP-Vis_Orange & NAC \\
24 & 480.7 & FFP-Vis_Blue & NAC \\
21 & 535.7 & Green_Empty & WAC \\
\hline
\end{tabular}

the first step is to derive the distance of the particles to the cameras. There are different methods that use a variety of data. Fulle et al. (2016) used the parallax effect caused by the spacecraft motion. They made the assumption that the dust moved radially away from the nucleus, which we show to be a reasonable assumption. Since the cameras were pointed towards the general direction of the centre of the nucleus, any apparent motion of the particles on consecutive frames was interpreted as due to parallax. Combining the spacecraft velocity, the exposure times and the observed particle movement yield the distance of the particles via parallax determinations. This method works with particles at a large distance range. Davidsson et al. (2015) also computed the distance of particles by using the parallax effect. They derived the orbits of four particles from consecutive images by determining the motion of the particles with respect to the stars. Güttler et al. (2017) derived the distance of particles close to the spacecraft via defocusing. Indeed, if the particles are close enough to the cameras their trails appear unfocused. From the degree of defocusing the distance can be derived.

We determined the distance of the particles from double-camera observations by taking advantage of the way the cameras are mounted on Rosetta, i.e. parallel optical axes and a baseline of $0.7 \mathrm{~m}$. If a particle is in a certain distance range from the spacecraft and recorded simultaneously by both cameras, this configuration allows the measurement of a shift between the trails seen by NAC and WAC. This is due to the parallax effect allowing the determination of the distance without any assumptions on the relative motion between particle and spacecraft. Once the distance of the particles is known, their sizes and masses can be computed, as well as their velocities. We found particles with diameters in the order of centimetres and masses in the range of $10^{-6}-10^{2} \mathrm{~kg}$. The mean velocity is found to be $2.4 \mathrm{~m} \mathrm{~s}^{-1}$ relative to the cometary nucleus. The data of the NAC were collected in different filters making it possible to derive the spectral slope. The value of about $11 \% / 100 \mathrm{~nm}$ at perihelion with a decreasing trend in time matches the results of Frattin et al. (2017). Furthermore, the number density of the particles can be computed as well as the dust mass-loss rate of the nucleus. The results are in good agreement with the values found by Fulle et al. (2016).

A value that can be derived from in situ as well as from groundbased observations is the coma brightness in terms of Af $\rho$ (A'Hearn et al. 1984). The evolution of $A f \rho$ for 67P with time and hence solar distance is presented by Boehnhardt et al. (2016). They used data collected with a $2 \mathrm{~m}$ telescope at Mt. Wendelstein Observatory, from 2015 August (just after perihelion) until 2016 May. Their fig. 4 shows a linear decreasing behaviour of Af $\rho$ with time starting at about $4 \mathrm{~m}$ just after perihelion. Snodgrass et al. (2017) state the Af $\rho$ peaking at $10 \mathrm{~m}$.

\section{THE DATA}

To observe the dynamics of the dust in the coma of the comet, multiple image sequences (called GRAIN-COLOR) were taken with both cameras, NAC and WAC, collecting data at the same time. The utilized data were collected around the perihelion of 67P (2015 August 13), between 2015 July 24 and 2015 September 28. The dates are listed in Table 1. The data could be combined into image sets of four or five single images. The WAC took one image with an exposure time of $64 \mathrm{~s}$ (before perihelion) or $90 \mathrm{~s}$ (after perihelion). Simultaneously, the NAC took three consecutive images in three different filters, F41, F22 and F24, each with an exposure time of 12.5 s. Given the comparatively long exposure times, the dust particles appear as trails on the images. After perihelion, the NAC sequence was expanded with one image in the orange filter (F32) with a near focus plate instead of the far focus plate used by F22. The filters 


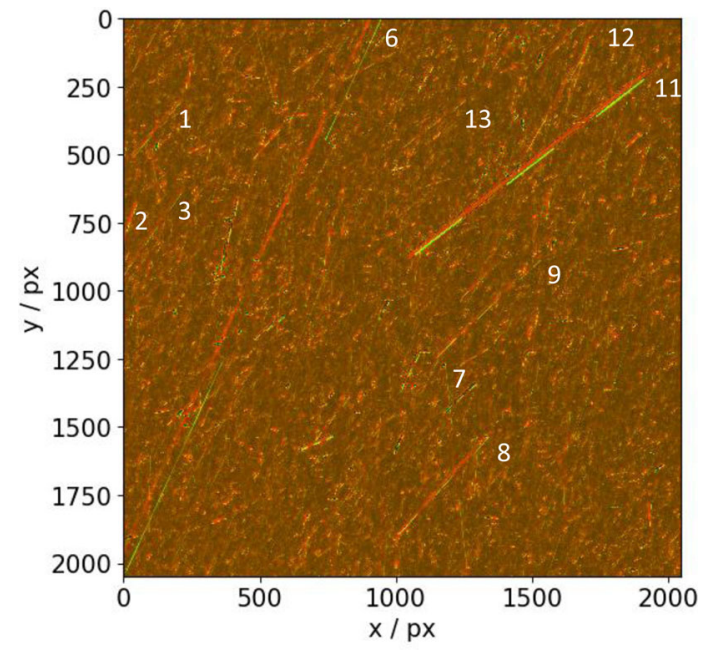

Figure 1. The data set collected 2015 August 4 at about 14:30 UTC shown as an aligned red-green stack. The WAC image is presented in red. The NAC maximum stack is shown in green. Note the three divided parts of the green trails. All particles for that a reliable distance determination could be carried out are marked with their indices. The names of the original images are WAC_2015_08_04T14_30_45_805Z_ID30_1397549700_F21， NAC_2015_ 08_04T14_30_48_822Z_ID30_1397549000_F41, NAC_2015_08_04T14_31_ 12_683Z_ ID30_1397549001_F22, NAC_2015_08_04 T14_31_36_ 689Z_ID30_1397549002_F24.

with their central wavelengths are listed in Table 2 . The utilized images are of level 3, meaning that a calibration was done based on radiometry and a correction was applied for geometric distortion. The OSIRIS image calibration is explained in Tubiana et al. (2015). In total, there are 40 image sets usable for the method explained here and hence including at least one particle with a shifted trail. This corresponds to a total number of 178 single images.

The main difference between the two cameras is their FOVs. Hence, for double-camera observations the WAC image has to be cropped to the dimensions of the NAC data. Moreover, the single images have to be aligned to correct for small changes in the pointing direction as well as for small changes most likely caused by small tremors of the spacecraft. These are small effects of only a few pixels but for the distance determination a difference of some pixels would yield significantly deviating results. This was prevented by using manually chosen reference stars to align the images with the software PIXINSIGHT (Pleiades Astrophoto S. L. 2017, 1.8, Valencia, Spain). The software rotates and shifts the images to align always with the first NAC image of a sequence, interpolating all other images. This was only carried out for the distance determination. For all other computations, the original data was used. An example of one aligned image set is presented in Fig. 1 as a red-green image. It shows 10 particles with visible parallax shifts between the green NAC and the red WAC data. They are marked with the particle indices used in this image set on their right-hand side. The data was recorded 2015 August 4 at about 14:30 UTC. The distances are listed in Table 3.

\section{METHOD}

\subsection{Distance determination}

If the particles are less than $6000 \mathrm{~m}$ from the spacecraft, they show a shift between the trail seen by the NAC and the trail recorded by the WAC. At distances larger than $6000 \mathrm{~m}$ the shift between the trails is too small to be identifiable. The distance determination method is explained in more detail in Drolshagen et al. (2017). In the image sets there are in total 262 particles for which a distance determination was possible.

A half-automatic particle detection programme was used to identify the start and end positions of the trails, see Ott et al. (2016). For very faint trails in combination with noisy images, the positions were determined by hand. The positioning accuracy was estimated based on the measurement technique: 1 pixel (automatic determination of both the NAC and the WAC trail), 1.2 pixel (automatic determination of the WAC trail), 1.3 pixel (automatic determination of the NAC trail) or 1.5 pixel (both trails were determined by hand). The errors on the distance were determined by propagating the maximum and minimum pixel shift. Since the shift for large distances is small, a shift error has a higher impact than on lower distances. Consequently, the uncertainty for larger distances is higher than for lower distances. We point out that we only consider a particle movement in the $y-x$ plane of the images during the exposure. The results of this method are presented in Section 4.1.

\subsection{Size determination}

Once we know the distances, we are able to derive the sizes and the masses of the particles. This is done with the method published by Güttler et al. (2017) using the commonly utilized density assumption of $\rho_{\text {aggregate }}=1000 \mathrm{~kg} \mathrm{~m}^{-3}$, extracted from the density range derived by Rotundi et al. (2015). They use a photometry analysis of the particles to find the reflectance and calculate the particle sizes

Table 3. List of the 10 analysed particles in the image set collected 2015 August 04 at about 14:30 UTC, compare Fig. 1. They can be identified with their index and the title of the WAC images of the corresponding set. Additionally, the determined distance of the particle is given as well as its integrated intensity. Furthermore, the velocity of the particle, its size and its mass is listed. A full list of all 262 analysed particles can be found online.

\begin{tabular}{|c|c|c|c|c|c|c|}
\hline Index & Name & Distance $(\mathrm{m})$ & Median reflectance & Velocity $\left(\mathrm{m} \mathrm{s}^{-1}\right)$ & Size $(\mathrm{m})$ & Mass $(\mathrm{kg})$ \\
\hline 2 & WAC_2015_08_04T14_30_45_805Z_ID30_1397549700_F21 & 4914 & $1.5 \mathrm{E}-03$ & $1.20 \mathrm{E}+00$ & $4.61 \mathrm{E}-02$ & $5.12 \mathrm{E}-02$ \\
\hline 6 & WAC_2015_08_04T14_30_45_805Z_ID30_1397549700_F21 & 809 & $5.7 \mathrm{E}-04$ & $2.29 \mathrm{E}+00$ & $2.51 \mathrm{E}-02$ & $8.23 \mathrm{E}-03$ \\
\hline 7 & WAC_2015_08_04T14_30_45_805Z_ID30_1397549700_F21 & 5378 & $3.1 \mathrm{E}-03$ & $4.58 \mathrm{E}+00$ & 7.37E-02 & $2.10 \mathrm{E}-01$ \\
\hline 8 & WAC_2015_08_04T14_30_45_805Z_ID30_1397549700_F21 & 3150 & $2.9 \mathrm{E}-03$ & $1.63 \mathrm{E}+00$ & $4.13 \mathrm{E}-02$ & $3.70 \mathrm{E}-02$ \\
\hline 9 & WAC_2015_08_04T14_30_45_805Z_ID30_1397549700_F21 & 4213 & $1.9 \mathrm{E}-03$ & $2.01 \mathrm{E}+00$ & $4.46 \mathrm{E}-02$ & $4.65 \mathrm{E}-02$ \\
\hline 12 & WAC_2015_08_04T14_30_45_805Z_ID30_1397549700_F21 & 3098 & $2.1 \mathrm{E}-03$ & $1.90 \mathrm{E}+00$ & $3.48 \mathrm{E}-02$ & $2.22 \mathrm{E}-02$ \\
\hline 13 & WAC_2015_08_04T14_30_45_805Z_ID30_1397549700_F21 & 3780 & $8.5 \mathrm{E}-04$ & $1.52 \mathrm{E}+00$ & $2.71 \mathrm{E}-02$ & $1.04 \mathrm{E}-02$ \\
\hline
\end{tabular}


assuming the same reflectance properties for the nucleus of $67 \mathrm{P}$ and the particles.

$s=\frac{2}{\sqrt{\pi}} \sqrt{\frac{R_{\text {dust }}}{R_{67 \mathrm{P}}} \frac{13.5 \times 10^{-6} \mathrm{~m}}{\mathrm{FL}} d_{\text {particle }} .}$

The size of the particles (see Formula 1) given by their diameter $s$ depends on the focal lengths, FL, of the cameras, which, for the calibrated images, corresponds to $0.717322 \mathrm{~m}$ for the NAC and $0.13568 \mathrm{~m}$ for the WAC. The calculation also includes the size of the pixels of the CCD which is $13.5 \times 10^{-6} \mathrm{~m}$, taken from Keller et al. (2007). $R_{\text {dust }}$ is the particle's integrated reflectance. $R_{67 \mathrm{P}}$ depends on the phase angle at which the image is taken. It is the reflectance of the surface of the nucleus recorded at this angle. $d_{\text {particle }}$ is the distance of the particle to the cameras.

We point out that our particles might be rotating and that we assume a spherical form. The sizes are determined for all trails that are completely included in the original images so that an integrated reflectance over the whole trail could be computed. If there were trails recorded in full by NAC and WAC, only the NAC trails were used. This is due to the larger uncertainty of the WAC data compared to the signals recorded by NAC because of the larger entrance pupil of the NAC. In most cases, the median size determined from the NAC trails is used. We were able to derive sizes for 256 particles. A size error was estimated using the error of the particle reflectance, utilizing the same method used by Güttler et al. (2017) and propagating the error. The size range can be found in Section 4.2.

\subsection{Spectral slope determination}

Due to the fact that the NAC took images consecutively in different filters the spectral slope of the particles could be investigated. A linear fit performed through these values shows an increase of the particle reflectance with increasing wavelength. This can be computed for all particles for which the NAC recorded data in all three filters. Because the F32, added after perihelion, also used the orange filter, and the integrated reflectance values collected with this filter were found to match the F22 values, the F32 values were not considered for this. In total, there are 130 particles for which a spectral slope could be determined.

The formula of the spectral slope from Frattin et al. (2017) is used, see equation (2), based on the work of Fornasier et al. (2015).

Spectral slope $=\frac{\frac{I}{F_{882}}-\frac{I}{F_{535}}}{\frac{I}{F_{535}}(882-535 \mathrm{~nm})} 10000$.

The object's radiance is given by $I$ and the solar flux by $F$. Hence, $I / F$ is the particle reflectance at the different filters.

With the fit through the reflectance values we calculated the integrated reflectance of the particle for $\lambda-353 \mathrm{~nm}$, which is the wavelength of the green filter. Fig. 2 shows the normalized integrated reflectance over the central wavelength of the used NAC filter for four randomly selected particles. They were normalized for the green filter values. The legend shows the reflectance each trail would have had at $535 \mathrm{~nm}$. The absolute reflectance values of the particles vary by an order of magnitude. The larger and closer a particle is the brighter is its signal. None the less, the particles all show a comparable trend of integrated reflectance over wavelength. The slope between 480 and $649 \mathrm{~nm}$ is in most cases larger than the one found in the range of 649 and $882 \mathrm{~nm}$. This trend is in agreement with the signals found by Frattin et al. (2017) and interpreted

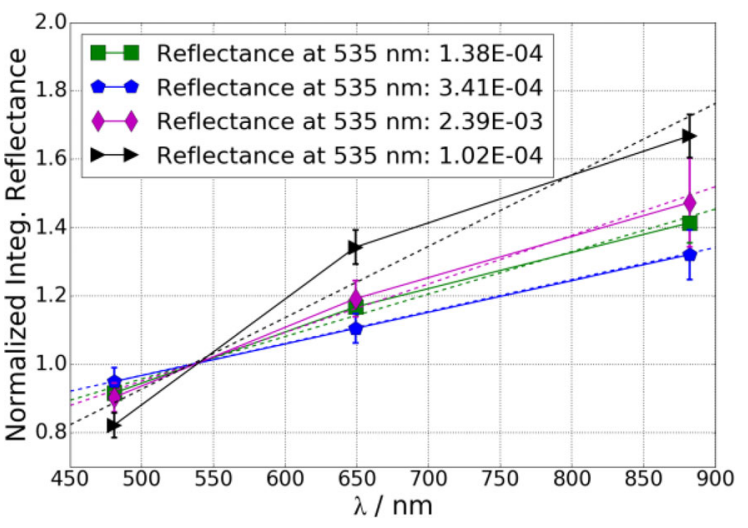

Figure 2. The normalized integrated reflectance of four randomly selected particles marked with different symbols and error values. They represent an overall trend observed in the data set. The increase in the reflectance can clearly be seen. In the legend the extrapolated reflectance value of the particles for $535 \mathrm{~nm}$ is given. The reflectance values of the single trails normalized to the green filter value are presented over the central wavelength of the used NAC filters with a linear fit. $\lambda=480.7 \mathrm{~nm}$ corresponds to F24, $\lambda=649.2 \mathrm{~nm}$ corresponds to F22 and $\lambda=882.1 \mathrm{~nm}$ corresponds to F41.

therein. The determined results of all spectral slopes can be found in Section 4.3.

\subsection{Velocity determination}

In the next step the velocity of the particles was computed. For all trails that are completely in the image the trail lengths in pixels were computed. With the trail length and the known exposure time, the velocity of a particle was computed. There are 257 particles for which a velocity determination was possible. We could assign a direction of movement for a subsample of 216 particles. Indeed, only particles with at least one full and one partial trail have enough information on the timing. If there is NAC and WAC information available again only the NAC data were used together with the mean velocity of the single NAC trails. In the images the velocity can be expressed in pixels per second. With the spatial resolution of the cameras $\left(0.0011^{\circ} \mathrm{pixel}^{-1}\right.$ for the NAC and $0.0059^{\circ} \mathrm{pixel}^{-1}$ for the WAC) and the known distance of the particles the velocity in $\mathrm{m} \mathrm{s}^{-1}$ can be computed. The measured raw velocities are corrected for two factors: first, the spacecraft movement, secondly the direction of the nucleus from which the particles come from. The second correction is required as the velocity of the particles found on the images is a projection of the true velocity to the plane perpendicular to the viewing direction of the cameras. The spacecraft motion can be subtracted from the apparent velocities, if the direction of the particles is known. Assuming that the particles all move radially away from the nucleus, knowing the angle between the nucleus and the pointing direction of the camera, the presumed particle movement can be computed. In this way, the apparent velocities can be corrected to derive the real particle velocities, estimating a velocity component in the $z$-direction. The found velocities are shown in Section 4.4.

\subsection{Dust mass-loss rate determination}

With the mass distribution, the number density $\rho_{\text {raw }}$ of the particles can be computed. This value is defined as the number of particles per $\mathrm{m}^{3}$ per image in a mass bin. The number of particles is given by the NAC trails. If a particle is included completely in only one of the 


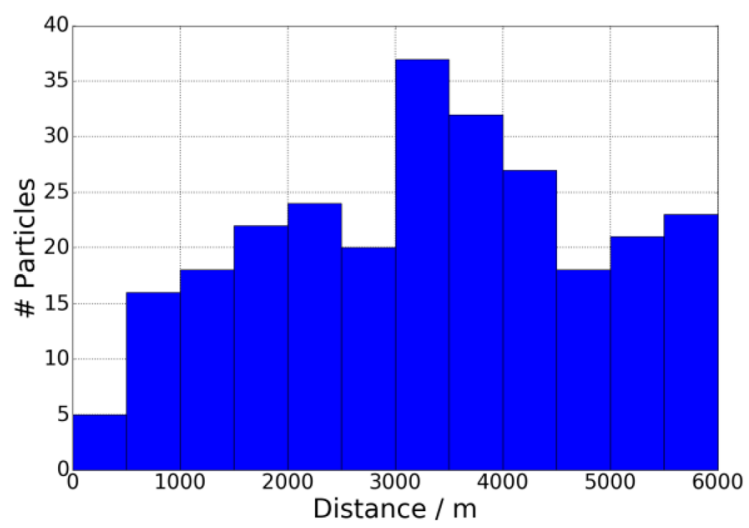

Figure 3. Particle-spacecraft distance distribution. The distances of 262 particles for that the distance to the spacecraft could be determined are presented.

consecutively taken NAC images it is only counted once. If it can be seen in three NAC images it is counted three times. If a trail leaves or enters the NAC FOV during the exposure and the trail is only partially visible in the image, it is counted half. It is assumed that the error occurring by not counting them proportionally to their trail lengths is compensated by the large statistics where larger portions of particle trails cancel out smaller portions. Furthermore, the image sets that were analysed but in which no particles with a trail shift of a suitable size were found, have to be added to the calculations as well. Some image sequences were too noisy to identify any particles, with or without shifts, these were omitted from the total image number. Next, the number of particles is divided by the total number of NAC images which was found to be 176 .

From the number density, the dust mass-loss rate $Q_{\mathrm{m}}$ of the surface of the comets nucleus can be derived as explained by Fulle et al. (2016).

$Q_{n}=2 \pi R^{2} v \rho$

$Q_{m}=m Q_{n}$,

$v$ is the mean velocity in each mass bin. For this only the corrected velocities were used under the assumption that the motion of the particles is radially away from the nucleus. $R$ is the distance between the nucleus and the spacecraft. Since the data analysed herein were collected on different days at different distances (compare Table 1), a particle-weighted reference Rosetta-nucleus distance was used $(R=270000 \mathrm{~m})$. For each mass bin the integrated dust number loss rate at the surface of the nucleus, $Q_{\mathrm{n}}$, can be computed with equation (3). After multiplying $Q_{\mathrm{n}}$ with $m$, the mean dust mass in each mass bin, the dust mass-loss rate can be computed. The resulting dust mass-loss rate is presented in Section 4.5.

\section{RESULTS}

\subsection{Distance distribution}

The parallax distance determination method works for distances up to $6000 \mathrm{~m}$, for larger distances the shift between the trails becomes too small. The derived particle distance distribution is presented in Fig. 3 and ranges from 192 to $5990 \mathrm{~m}$ distance to the spacecraft. It shows a relatively uniform distribution. The five particles having a distance smaller than $500 \mathrm{~m}$ show a defocused signal, see Güttler et al. (2017), and hence the results of the distance have larger

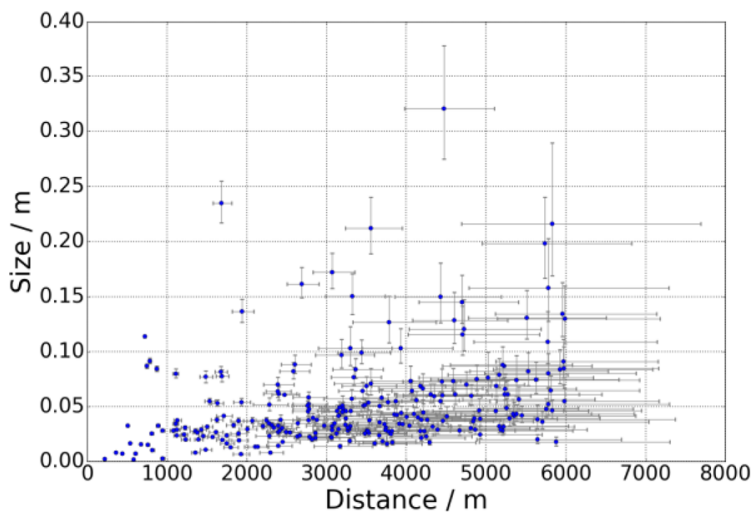

Figure 4. The size over the distance for 256 particles. The particles diameter in metre is shown over the distance of the particle. Presented in grey are the errors of the distance and size determinations.

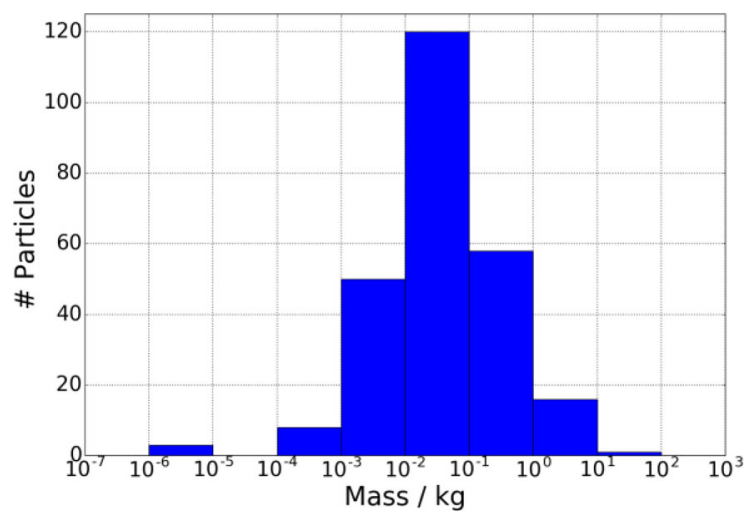

Figure 5. Histogram of the particle masses in $\mathrm{kg}$.

uncertainties. The mean distance is $3300 \mathrm{~m}$. It has to be kept in mind that due to the inverse square law, particles of the same size appear brighter, the closer they are to the cameras. Thus, a very small particle can only be detected if it is close to the spacecraft.

\subsection{Size distribution}

Of the 262 particles, six leave the NAC FOV during the exposure time. Five of them also leave the WAC FOV. The sixth particle's signal was so faint and noisy in the WAC data that the intensity determination did not yield a reliable result. Consequently, 256 particles are left for which sizes and masses could be determined.

Fig. 4 presents the sizes of the particles over their distances with the corresponding errors. The observed particles are found to have a diameter of some centimetres. The trend of detecting smaller particles closer to the spacecraft can be clearly seen. The mean particle size is $0.05 \mathrm{~m}$ with the smallest size of $1.5 \times 10^{-3} \mathrm{~m}$ and the largest size of $0.32 \mathrm{~m}$. Fig. 5 shows the mass distribution. It ranges from $10^{-6}-10^{2} \mathrm{~kg}$. It has to be noted that in the two smallest mass bins there were only three, respectively zero, particles found. In the largest mass bin there was only one particle detected. The mean mass is found to be $0.3 \mathrm{~kg}$, the smallest particle has a mass of $2 \times 10^{-6} \mathrm{~kg}$ and the largest one has a mass of $17 \mathrm{~kg}$. This is the same mass range as observed by Fulle et al. (2016) for particles in the coma of $67 \mathrm{P}$ around perihelion. 


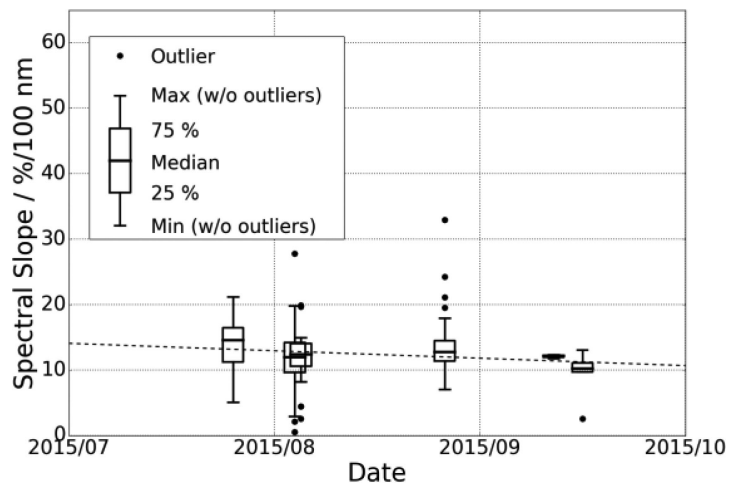

Figure 6. The spectral slope for the 130 particles for which it could be determined over the date of the observation. Each box plot shows the data of one observation day. The band in the box indicates the median of a day. Through those median values a linear fit is made (dashed line) which hints a tendency of decrease of the spectral slope in time. For the penultimate day, the spectral slope could only be computed for three particles, this value should be treated with caution.

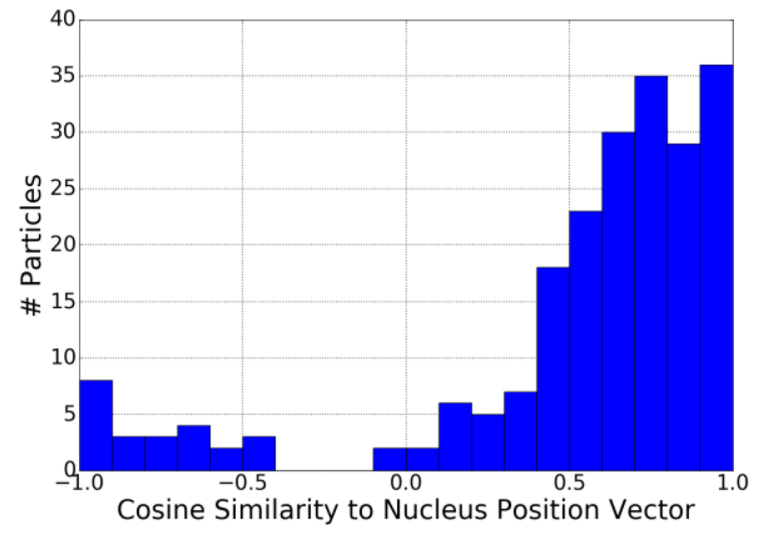

Figure 7. Histogram of the cosine similarity between the nucleus position vector and the particle movement vector. The closer the value is to 1 the more similar are the vectors.

\subsection{Spectral slope}

For all particles with information about the reflectance values of the different filters the spectral slope could be computed. They are presented in Fig. 6 plotted as blue circles over the observation day. For every day the median of the values is computed and presented as a diamond. Through those a linear fit is made and hints a tendency of decrease of the spectral slope in time. At perihelion the spectral slope derived with the fit is about $11 \% / 100 \mathrm{~nm}$. All these points are in agreement with the results found by Frattin et al. (2017).

\subsection{Velocity distribution}

The velocity distribution in the images is found to be relatively uniform. The mean velocity seen in the images is about 11 pixels s $\mathrm{s}^{-1}$. Taking into account the known distance of the particles, the mean apparent velocity is found to be about $0.6 \mathrm{~m} \mathrm{~s}^{-1}$. After correcting for the spacecraft motion this value increased to about $1 \mathrm{~m} \mathrm{~s}^{-1}$. As a following step, a radial motion away from the nucleus is assumed and used to correct the apparent velocity to the true velocity. We checked the particles to determine their directions and compared them to the position of the nucleus. The cosine similarity, giving the cosine of the angle between two vectors, of the velocity vector of

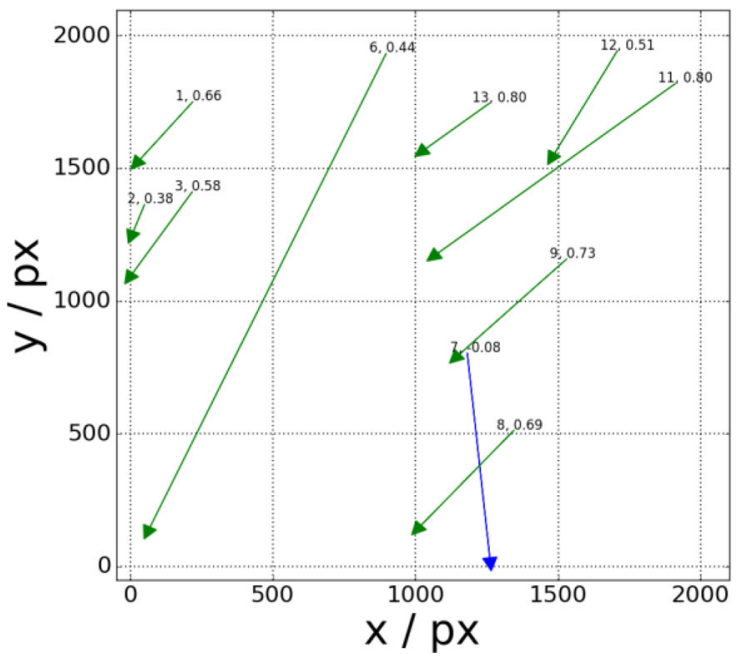

Figure 8. One example image with the particle directions drawn in as arrows and the particle index and the cosine similarity between the nucleus position vector and the particle movement vector. Included are the particles of Fig. 1.

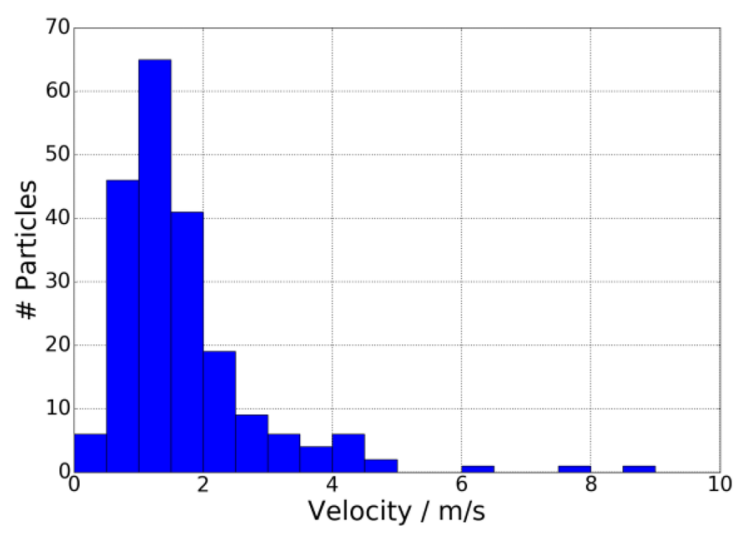

Figure 9. The velocity distribution of 207 of the 216 particles for which the velocity values could be corrected. Nine particles were faster than 10 $\mathrm{m} \mathrm{s}^{-1}$ and have values up to almost $30 \mathrm{~m} \mathrm{~s}^{-1}$.

the particle and the position vector of the nucleus is an indication for the radial motion of the particles. To compute this value the velocity values corrected for the spacecraft motion were used. Hence, only the $x$ - and $y$-directions can be compared. We found most of the cosine similarities to be higher than 0.5 (compare Fig. 7) which shows they are at least coming in general from a direction close to the nucleus. About 71 per cent of the particles have a cosine similarity value larger than 0.5 . This is a strong indication that they move radial away from the nucleus of 67P. None the less, about 12 per cent of the particles have a negative cosine similarity value and hence, do definitely not come directly from the nucleus. These particles and their orbits are an interesting aspect for future work. One example of derived particle directions is shown in Fig. 8, for the same particles as in Fig. 1. Furthermore, it was checked if there is a dependence of the sizes of the particles on their velocity and no dependence was found.

With the assumption of radial motion, the true particle velocity increases to a mean value of about $2.4 \mathrm{~m} \mathrm{~s}^{-1}$ relative to the nucleus. The velocity distribution is presented in Fig. 9 and shows 207 of the 
216 particles for which a velocity could be computed and that have a velocity slower or equal to $10 \mathrm{~m} \mathrm{~s}^{-1}$. The maximal velocity found is about $29.7 \mathrm{~m} \mathrm{~s}^{-1}$ and the minimal is about $0.3 \mathrm{~m} \mathrm{~s}^{-1}$. None the less, over 90 per cent of our velocity values have a velocity between 0 and $5 \mathrm{~m} \mathrm{~s}^{-1}$. Rotundi et al. (2015) observed particles from a comparable Rosetta-nucleus distance and found particles with velocities from 1 to $10 \mathrm{~m} \mathrm{~s}^{-1}$. However, they analysed data collected at $3.6 \mathrm{au}$ inbound from Sun. None the less, their median radial velocity was $3.5 \mathrm{~m} \mathrm{~s}^{-1}$ which is in good agreement with our $2.4 \mathrm{~m} \mathrm{~s}^{-1}$ mean velocity. Fulle et al. (2016) observed particles around the comet, i.e. at about 2.2 au inbound and at perihelion and found particle masses in the same mass range as we found. However, even though their particle velocities observed at $2.2 \mathrm{au}$ are smaller than ours (around $1.5 \mathrm{~m} \mathrm{~s}^{-1}$ ), their velocities at perihelion are larger (about $10 \mathrm{~m} \mathrm{~s}^{-1}$ ). This velocity discrepancy shows that our method could be biased towards slower particles.

All the particle properties mentioned in the previous sections were determined for each particle if the necessary information were available. In Table 3, 10 analysed particles from the image set collected 2015 August are listed with their properties. They can be identified with their index and the title of the WAC image of the corresponding set. Additionally, the determined distance of the particle is given as well as its integrated reflectance. Furthermore, the corrected velocity of the particle, its size and its mass are listed. A complete list of all our particles with their properties can be found online.

\subsection{Dust mass-loss rate}

The data analysed for this work were collected on different days with different observing conditions, varying the phase angle or the distance to the nucleus (compare Table 1). It was checked if there is a dependence of the sizes of the particles on the geometric parameters of observation but no dependence was found.

There is, however, a dependence on the particle size. Small particles are faint and can only be detected if they are close to the spacecraft. Hence, the size distribution shows a bias towards larger particles. This can be clearly seen in Fig. 4 in the lack of smaller particles at increasing distance from the spacecraft. To correct for this, a debiasing was carried out. For all distance bins the particle with the smallest size was taken and a linear fit was drawn through those, resulting in the detection limit stated in Formula (5). In the formula $d$ has to be inserted in metres. At $6000 \mathrm{~m}$ this fit yields a threshold size of about $0.026 \mathrm{~m}$. Consequently, all particles with a diameter larger than $0.026 \mathrm{~m}$ should be detected at all distances in the observation volume. However, the distribution can still be biased, e.g. faint particles near the detection limit in a very noisy image or at large phase angles were still not seen.

$S_{\text {smallest }}(d)=4.15 \times 10^{-6} d+1.02 \times 10^{-3}$.

For the number of particles per size bin observed per $\mathrm{m}^{3}$ only the observation volume up to a distance $d$ at which the particles of a certain size can be seen was used following equation (5). Formula (6) describes the computation of the observation volume up to a certain distance. According to the results of equation (5) for all particles larger than $0.026 \mathrm{~m}$ up to $d=6000 \mathrm{~m}$ the complete integrated observation volume of $1.07 \times 10^{8} \mathrm{~m}^{3}$ can be used. Formula (6) was derived by assuming the cameras to be at the same position

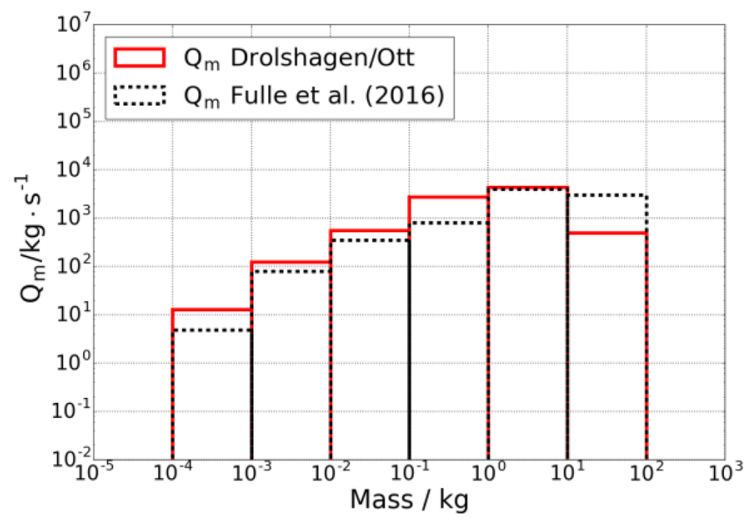

Figure 10. Dust mass-loss rate. The results achieved with the distance determination via parallax are shown as the solid line and the ones published by Fulle et al. (2016) as the dashed line.

and assuming a pyramid shape in space covered by the FOV of the NAC.

$V(d)=\frac{d}{3}\left(2 d \tan \left(\frac{2.22^{\circ}}{2}\right)\right)\left(2 d \tan \left(\frac{2.20^{\circ}}{2}\right)\right)$.

For every mass bin the mean mass, $m$, the mean dust cross-section, $\sigma$, as well as the mean velocity, $v$, is computed, see Table 4 . The number density $\rho_{\text {raw }}$ is determined and the debiased values $\rho$ are presented in the table. Furthermore, the dust number loss rate from the surface of the nucleus integrated in each mass bin, $Q_{\mathrm{n}}$, as well as the dust mass-loss rate at the surface, $Q_{\mathrm{m}}$, are listed. These values are also given by Fulle et al. (2016) for data collected around perihelion and hence comparable to the data used for this work. Comparing the number density values to the ones of Fulle et al., we see that our values are about one order of magnitude larger than the ones listed in their table 8 . Additionally, our velocity values are about a factor of 10 smaller than theirs. This yields comparable dust loss rates with Formula (3) and (4). The dust mass-loss rate computed with the method presented herein as well as the one published in Fulle et al. (2016) are shown in Fig. 10. The largest mass bin has to be considered with caution due to the lack of statistics in this mass range. Additionally, the smallest mass bin from $10^{-6}-10^{-5}$ $\mathrm{kg}$ is lost during the process of debiasing because all particles are smaller than the detection limit following Formula (5). None the less, it can be seen that the dust mass-loss rate is generally larger but in the same order as the ones found by Fulle et al. (2016). The summed-up value of $Q_{\mathrm{m}}$ is $8297 \mathrm{~kg} \mathrm{~s}^{-1}$.

This agreement results from a combination of effects. Our number densities are higher while our velocities are lower when compared to the values by Fulle et al. A combination of these two differences leads to very similar total dust mass-loss rates. It should be kept in mind that the observation conditions were different. Furthermore, the contribution to the coma brightness from each mass bin can be computed with the formula of Fulle et al. (2016), as stated in equation (7). For our data the geometric albedo, $A_{\mathrm{p}}$, is 6.5 per cent (Fornasier et al. 2015). As before, $Q_{\mathrm{n}}$ is the dust number loss rate of each mass bin, and $v$ is the corrected mean velocity of each mass bin. $\sigma$ is the mean dust cross-section of each mass bin, see Table 4. Integrating Af $\rho$ over all mass bins yields a value that can be compared to ground-based observations.

$A f \rho=\sum 2 A_{p} Q_{n} \sigma v^{-1}$. 
Table 4. Dust size distribution. For every mass bin, the mean mass per mass bin, $m$, the mean dust cross-section, $\sigma$, as well as the mean velocity, $v$, is given. The debiased number density, $\rho$ (the number of particles per image and per volume) is listed for every mass bin. The dust number loss rate from the surface of the nucleus integrated in each mass bin as well as the dust mass-loss rate at the surface are listed for every mass bin. Moreover, Af $\rho$ is presented, giving a value for the contribution to the coma brightness from each mass bin. The total Af $\rho$ is $6.8 \mathrm{~m}$.

\begin{tabular}{lccccccc}
\hline$\Delta m(\mathrm{~kg})$ & $m(\mathrm{~kg})$ & $\sigma\left(\mathrm{m}^{2}\right)$ & $v\left(\mathrm{~m} \mathrm{~s}^{-1}\right)$ & $\rho\left(\mathrm{m}^{-3}\right)$ & $Q_{\mathrm{n}}\left(\mathrm{s}^{-1}\right)$ & $Q_{\mathrm{m}}\left(\mathrm{kg} \mathrm{s}^{-1}\right)$ & $A f \rho(\mathrm{m})$ \\
\hline $10^{-4}-10^{-3}$ & $2.97 \mathrm{E}-04$ & $5.09 \mathrm{E}-05$ & $2.47 \mathrm{E}+00$ & $3.77 \mathrm{E}-08$ & $4.28 \mathrm{E}+04$ & $1.27 \mathrm{E}+01$ & $1.14 \mathrm{E}-01$ \\
$10^{-3}-10^{-2}$ & $5.14 \mathrm{E}-03$ & $3.34 \mathrm{E}-04$ & $2.67 \mathrm{E}+00$ & $1.96 \mathrm{E}-08$ & $2.40 \mathrm{E}+04$ & $1.23 \mathrm{E}+02$ & $3.91 \mathrm{E}-01$ \\
$10^{-2}-10^{-1}$ & $3.39 \mathrm{E}-02$ & $1.15 \mathrm{E}-03$ & $2.19 \mathrm{E}+00$ & $1.63 \mathrm{E}-08$ & $1.64 \mathrm{E}+04$ & $5.55 \mathrm{E}+02$ & $1.11 \mathrm{E}+00$ \\
$10^{-1}-10^{-0}$ & $2.75 \mathrm{E}-01$ & $4.69 \mathrm{E}-03$ & $2.79 \mathrm{E}+00$ & $7.86 \mathrm{E}-09$ & $1.01 \mathrm{E}+04$ & $2.77 \mathrm{E}+03$ & $2.20 \mathrm{E}+00$ \\
$10^{-0}-10^{-1}$ & $2.52 \mathrm{E}+00$ & $2.05 \mathrm{E}-02$ & $1.65 \mathrm{E}+00$ & $2.28 \mathrm{E}-09$ & $1.72 \mathrm{E}+03$ & $4.34 \mathrm{E}+03$ & $2.78 \mathrm{E}+00$ \\
$10^{-1}-10^{-2}$ & $1.73 \mathrm{E}+01$ & $8.08 \mathrm{E}-02$ & $1.13 \mathrm{E}+00$ & $5.56 \mathrm{E}-11$ & $2.88 \mathrm{E}+01$ & $4.97 \mathrm{E}+02$ & $2.67 \mathrm{E}-01$ \\
\hline
\end{tabular}

For the method presented herein the integrated $A f \rho$ value is about $6.9 \mathrm{~m}$. This is in the same order of magnitude as derived for ground-based observations (Boehnhardt et al. (2016), Snodgrass et al. 2017).

\section{CONCLUSIONS}

The scientific camera system on board Rosetta (OSIRIS) was mapping the Jupiter-family comet 67P/Churyumov-Gerasimenko for over 2 yr. With its two cameras the NAC and the WAC some image sequences were taken with both cameras working simultaneously. The data analysed herein were collected around 2015 August 13, the perihelion of 67P.

With the double-camera observations it was possible to determine the distance of the particles to the spacecraft via parallax. The cameras are mounted on Rosetta with a distance of about $0.7 \mathrm{~m}$ from each other. If the particles are less than $6000 \mathrm{~m}$ away from the spacecraft the trails seen by NAC and WAC display a shift between them. With this shift, the distance of the particle to the spacecraft can directly be computed. It was shown that the distance determination based on the double-camera observations works and needs only a few assumptions. The presented distance distribution shows a relatively uniform behaviour and includes 262 particles.

If the distance of the particles is known, it is possible to determine their sizes and masses. They are in good agreement with the values determined by Fulle et al. (2016) for the same distance of the nucleus to Sun. Furthermore, it was possible to derive the velocities of the particles and it was confirmed that most particles move nearly radially away from the comet. The particles that do not come radially from the nucleus and their orbits are an interesting aspect for future work.

Additionally, the analysed data of the NAC were taken with different filters. This allowed us to derive a spectral slope comparable to the ones of Frattin et al. (2017). We found that the spectral slope decreases with time which is in agreement with the values of Frattin et al.

Moreover, it was possible to derive the number density of the particles, i.e. the number of particles per image per $\mathrm{m}^{3}$ and per mass bin. From this the dust mass-loss rate of $67 \mathrm{P}$ could be determined. The results are in agreement with the ones published by Fulle et al. (2016). Furthermore, an Af $\rho$ was derived, in the same order of magnitude as ground-based observations found for 67P around perihelion (Boehnhardt et al. 2016; Snodgrass et al. 2017).

\section{ACKNOWLEDGEMENTS}

OSIRIS was built by a consortium led by the Max-PlanckInstitut für Sonnensystemforschung, Göttingen, Germany, in collaboration with Centro di Ateneo di Studi ed Attivit'a Spaziali 'Giuseppe Colombo' (CISAS), University of Padova, Italy; the Laboratoire d'Astrophysique de Marseille, France; the Instituto de Astrofísica de Andalucia, Consejo Superior de Investigaciones Científicas (CSIC), Granada, Spain; the Science Support Office of the European Space Agency, Noordwijk, the Netherlands; the Instituto Nacional de Técnica Aeroespacial, Madrid, Spain; the Universidad Politéchnica de Madrid, Spain; the Department of Physics and Astronomy of Uppsala University, Sweden and the Institut für Datentechnik und Kommunikationsnetze der Technischen Universität Braunschweig, Germany.

Rosetta is an ESA mission with contributions from its member states and NASA. The support of the national funding agencies of Germany (DLR), France (CNES), Italy (ASI), Spain (MEC), Sweden (SNSB) and the ESA Technical Directorate is gratefully acknowledged.

We thank the Rosetta Science Ground Segment at ESAC, the Rosetta Mission Operations Centre at ESOC and the Rosetta Project at ESTEC for their outstanding work enabling the science return of the Rosetta Mission.

\section{REFERENCES}

A'Hearn M. F., Schleicher D. G., Millis R. L., Feldman P. D., Thompson D. T., 1984, AJ, 89, 579

Boehnhardt H., Riffeser A., Kluge M., Ries C., Schmidt M., Hopp U., 2016, MNRAS, 462, 376

Davidsson B. J. et al., 2015, A\&A, 583, 16

Drolshagen E. et al., 2017, Planet. Space Sci., 143, 256

Fornasier S. et al., 2015, A\&A, 583, A30

Frattin E. et al., 2017, MNRAS, in press

Fulle M. et al., 2016, AJ, 821, 19

Güttler et al., 2017, MNRAS, in press

Keller H. U. et al., 2007, Space Sci. Rev., 128, 433

Ott T., Drolshagen E., Koschny D., Poppe B., 2016, in Roggemans A., Roggemans P., eds, The Particle Detection Program. Proceedins of the international Meteor Conference, Egmond, the Netherlands, p. 209

Pleiades Astrophoto S. L., 2017, PixInsight. Available at: http://pixinsight.com/

Rotundi A. et al., 2015, Science, 347, aaa3905

Snodgrass C., A'Hearn M. F., Aceituno F., Afanasiev V., 2017, Phil. Trans. R. Soc. A, 375, 20160249

Tubiana C. et al., 2015, A\&A, 583, A46

${ }^{1}$ University of Oldenburg, Ammerländer Heerstraße 114, D-26111 Oldenburg, Germany

${ }^{2}$ ESA/ESTEC, Keplerlaan 1, NL-2201 AZ Noordwijk ZH, the Netherlands

${ }^{3}$ Chair of Astronautics at TU Munich, Arcisstraße 21, D-80333 Munich, Germany

${ }^{4}$ Max Planck Institute for Solar System Research, Justus-von-Liebig-Weg, 3, D-37077 Göttingen, Germany 


\section{S284 T. Ott et al.}

${ }^{5}$ INAF, Osservatorio Astronomico di Padova, Vicolo dell'Osservatorio 5, I-35122 Padova, Italy

${ }^{6}$ Dipartimento di Fisica e Astronomia 'G.Galilei', University of Padova, Vicolo dell'osservatorio 3, I-35122 Padova, Italy

${ }^{7}$ Department of Physics and Astronomy, University of Padova, Vicolo dell' Osservatorio 3, I-35122 Padova, Italy

${ }^{8}$ Laboratoire d'Astrophysique de Marseille, UMR 7326, CNRS \& Aix Marseille Université, F-13388 Marseille Cedex 13, France

${ }^{9}$ Centro de Astrobiologia, CSIC-INTA, E-28850 Madrid, Spain

${ }^{10}$ International Space Science Institute, CH-3012 Bern, Switzerland

${ }^{11}$ Department of Physics and Astronomy, Uppsala University, Box 516, SE75120 Uppsala, Sweden

${ }^{12}$ PAS Space Research Center, Bartycka 18A, PL-00716 Warszawa, Poland ${ }^{13}$ Department of Astronomy, University of Maryland, College Park, MD 20742, USA

${ }^{14}$ Jet Propulsion Laboratory, M/S 183-301, 4800 Oak Grove Drive, Pasadena, CA 91109, USA

${ }^{15}$ LESIA, Observatoire de Paris, PSL Research University, CNRS, Univ. Paris Diderot, Sorbonne Paris Cité, UPMC Univ. Paris 06, Sorbonne Universités, 5 place Jules Janssen, F-92195 Meudon, France

${ }^{16}$ LATMOS, CNRS/UVSQ/IPSL, 11 Boulevard d'Alembert, F-78280 Guyancourt, France

${ }^{17}$ CNR-IFN UOS Padova LUXOR, Via Trasea 7, I-35131 Padova, Italy

${ }^{18}$ Department of Industrial Engineering, University of Padova, Via Venezia 1, I-35131 Padova, Italy

${ }^{19}$ University of Trento, via Sommarive 9, I-38123 Trento, Italy

${ }^{20}$ INAF, Osservatorio Astronomico di Trieste, via Tiepolo 11, I-34143 Trieste, Italy

${ }^{21}$ Operations Department, European Space Astronomy Centre/ESA, P.O.

Box 78, E-28691 Villanueva de la Canada, Madrid, Spain
${ }^{22}$ Aix Marseille Université, CNRS, Laboratoire d'Astrophysique de Marseille, UMR 7326, F-13388 Marseille, France

${ }^{23}$ Instituto de Astrofisica de Andalucia (CSIC), c/ Glorieta de la Astronomia s/n, E-18008 Granada, Spain

${ }^{24}$ Deutsches Zentrum für Luft- und Raumfahrt (DLR), Institut für Planetenforschung, Rutherfordstrasse 2, 12489 Berlin, Germany

${ }^{25}$ Graduate Institute of Astronomy, National Central University, 300 ChungDa Rd, Chung-Li 32054, Taiwan

${ }^{26}$ Space Science Institute, Macau University of Science and Technology, Macao, China

${ }^{27}$ Institut für Geophysik und extraterrestrische Physik, Technische Universität Braunschweig, Mendelssohnstrasse 3, D-38106 Braunschweig, Germany

${ }^{28}$ Centro di Ateneo di Studi ed Attività Spaziali 'Giuseppe Colombo' (CISAS), University of Padova, via Venezia 15, I-35131 Padova, Italy

${ }^{29}$ Department of Information Engineering, University of Padova, I-3513I Padova, Italy

${ }^{30}$ NASA Ames Research Center, Moffett Field, CA 94035, USA

${ }^{31}$ Physikalisches Institut, University of Bern, Sidlerstrasse 5, CH-3012 Bern, Switzerland

${ }^{32}$ Center for Space and Habitability, University of Bern, CH-3012 Bern, Switzerland

This paper has been typeset from a $\mathrm{TE}_{\mathrm{E}} \mathrm{X} / \mathrm{L} \mathrm{T} \mathrm{E} \mathrm{X}$ file prepared by the author. 